\title{
CRISPR/Cas9 targeting of GPRC6A suppresses prostate cancer tumorigenesis in a human xenograft model
}

\author{
Ruisong Ye ${ }^{1}$, Min $\mathrm{Pi}^{1 *}$, John V. Cox², Satoru K. Nishimoto ${ }^{2}$ and L. Darryl Quarles ${ }^{1 *}$
}

\begin{abstract}
Background: GPRC6A is implicated in the pathogenesis of prostate cancer, but its role remains uncertain because of a purported tolerant gene variant created by substitution of a K..Y polymorphism in the 3rd intracellular loop (IL) that evolved in the majority of humans and replaces the ancestral RKLP present in $40 \%$ of humans of African descent and all other species.

Methods: We determined whether the K..Y polymorphism is present in human-derived prostate cancer cell lines by sequencing the region of the 3rd IL and assessed the cellular localization of a "humanized" mouse GPRC6A containing the K..Y sequence by immunofluorescence. We assessed functions of GPRC6A in PC-3 cells expressing endogenous GPRC6A and in GPRC6A-deficient PC-3 cells created using CRISPR/Cas9 technology. The effect of GPRC6A on basal and ligand stimulated cell proliferation and migration was evaluated in vitro in wild-type and PC-3-deficient cell lines. The effect of editing GPRC6A on prostate cancer growth and progression in vivo was assessed in a Xenograft mouse model implanted with wild-type and PC-3 deficient cells and treated with the GPRC6A ligand osteocalcin.
\end{abstract}

Results: We found that all of the human prostate cancer cell lines tested endogenously express the "K.Y" polymorphism in the 3rd IL. Comparison of mouse wild-type GPRC6A with a "humanized" mouse GPRC6A construct created by replacing the "RKLP" with the "K..." sequence, found that both receptors were predominantly expressed on the cell surface. The transfected "humanized" GPRC6A receptor, however, preferentially activated mTOR compared to ERK signaling in HEK-293 cells. In contrast, in PC-3 cells expressing the endogenous GPRC6A with the "K.Y" polymorphism, the ligand osteocalcin stimulated ERK, AKT and mTOR phosphorylation, promoted cell proliferation and migration, and upregulated genes regulating testosterone biosynthesis. Targeting GPRC6A in PC-3 cells by CRISPR/Cas9 significantly blocked these responses in vitro. In addition, GPRC6A deficient PC-3 xenografts exhibited significantly less growth and were resistant to osteocalcin-induced prostate cancer progression compared to control PC-3 cells expressing GPRC6A.

Conclusions: Human GPRC6A is a functional osteocalcin and testosterone sensing receptor that promotes prostate cancer progression. GPRC6A may contribute to racial disparities in prostate cancer, and is a potential therapeutic target to develop antagonists to treat prostate cancer.

Keywords: Osteocalcin, GPRC6A, CRISPR/Cas9, Prostate cancer

\footnotetext{
*Correspondence: mpi@uthsc.edu; dquarles@uthsc.edu

'Department of Medicine, University of Tennessee Health Science Center,

19 S Manassas St., Memphis, TN 38163, USA

Full list of author information is available at the end of the article
} 


\section{Background}

Prostate cancer is the most commonly diagnosed cancer and the second leading cause of cancer-related deaths in men in the United States [1]. Androgen depravation therapy (ADT) is the initial treatment for this disorder, but the disease often progresses to more clinically aggressive castrate-resistant prostate cancer (CRPC). There is an unmet need to understand the molecular pathogenesis of CRPC and identify novel pharmacological targets to design new treatments for prostate cancer [2].

GPRC6A is a widely expressed G-protein coupled receptor that is a master regulator of energy metabolism, and sexual reproduction [3-13]. Ligands for GPRC6A include osteocalcin (Ocn), a bone derived hormone [15], testosterone $(\mathrm{T})$, basic amino acids, such as Larginine (L-Arg), and cations, such as calcium and zinc [16]. While testosterone can be converted to DHT to activate androgen receptors, and other sensing mechanisms exist for L-Arg [17], and cations [16], GPRC6A is the only identified receptor for osteocalcin [5]. GPRC6A is expressed in multiple mouse tissues, where it regulates metabolic processes and stimulates the release of hormones, including insulin from $\beta$-cells $[3$, 18], testosterone from Leydig cells [6], glucagon-like peptide-1 (GLP-1) from intestinal cells $[7,8]$, adiponectin from adipocytes $[9,19]$, and interleukin 6 (IL-6) from myocytes [10].

There is emerging evidence that GPRC6A is involved in the pathogenesis of prostate cancer. First, GPRC6A is increased in human prostate cancer cell lines and in a subset of human prostate cancer tumors [12]. Second, activation of GPRC6A stimulates cell proliferation and chemotaxis in human prostate cancer cells [12], and promotes prostate cancer epithelial-mesenchymal transition [20], whereas knockdown of GPRC6A inhibits prostate cancer cell migration and invasion [12]. Third, human genome wide association studies (GWAS) link single nucleotide polymorphisms (SNPs) in the GPRC6A locus with the development of prostate cancer in the Japanese, and Chinese population [21-23]. More recently, the SNP rs1606365 GPRC6A was found to be associated with aggressive prostate cancer [20]. Fourth, genetic ablation of GPRC6A attenuates prostate cancer progression in the TRansgenic Adenocarcinoma of the Mouse Prostate (TRAMP) model [12], Fifth, high serum levels of osteocalcin is a biomarker of prostate cancer [24], and the autocrine/paracrine release of osteocalcin may stimulate prostate cancer growth and progression through activation of GPRC6A [25]. Finally, GPRC6A mediates the non-genomic, rapid signaling responses to testosterone [26], leading to activation of PI3K/Akt pathways that are implicated in androgen receptor (AR)independent progression and therapeutic resistance of prostate cancer [27]. GPRC6A may provide a molecular basis for the association between Metabolic Syndrome and the risk of prostate cancer [14].

A direct role of GPRC6A in the pathogenesis of human prostate cancer, however, remains to be established with certainty. Some observations even suggest that evolutionary divergence of GPRC6A in humans has led to a loss-of-function tolerant gene variant with little pathophysiological effects [28-30]. GPRC6A is not identified in gene expression signatures that predict prostate cancer disease severity in humans [31]. Moreover, questions regarding the functions GPRC6A and its ligands have been raised [28, 29, 32]. Finally, intracellular retention of human GPRC6A has been reported [29, 33], suggesting that the human GPRC6A receptor may have diminished cell surface expression and function.

In the current study, we examined the function of human GPRC6A in prostate cancer progression in vitro and in vivo. We demonstrated that GPRG6A is expressed in human prostate cancer cell lines and has an evolutionarily divergent polymorphism that does not affect cell surface expression but preferentially enhances mTOR signaling. We also used clustered regularly interspaced short palindromic repeats (CRISPR) and CRISPR-associated protein 9 nuclease (Cas9) (CRISPR/Cas9) [34] to disrupt the GPRC6A gene in the human prostate cancer cell line (PC-3). We found that editing the endogenous GPRC6A gene using CRISPR/Cas9 inhibits osteocalcin activation of ERK, AKT, and mTOR signaling pathways, and cell proliferation and migration in vitro. Finally, we found that GPRC6A mediates prostate cancer progression in vivo by assessing the response to osteocalcin in human prostate cancer xenograft models of cells expressing endogenous GPRC6A or with CRISPR/Cas9 mediated deletion of GPRC6A. Taken together, our findings support a role for GPRC6A and osteocalcin in prostate cancer, and define a potential therapeutic target to suppress prostate cancer progression.

\section{Methods \\ Cell culture and reagents}

Human prostate cancer cell lines, PC-3, DU145, LNCap and 22Rv1 were cultured in the RMPI1640 medium (Life Technologies, \#A10491-01) supplemented with 10\% FBS (ATLANTA biologicals; S11150), 10 units/ml penicillin, and $100 \mu \mathrm{g} / \mathrm{ml}$ streptomycin (Life Technologies; 15140).

Decarboxylated osteocalcin was produced and purified as described previously [5].

Human GPRC6A cDNA was RT-PCR-amplified from human kidney total RNA and cloned into the expression vector pcDNA3.1(+)-Puro as previously described [18]. Mouse GPRC6A cDNA construct was a gift from Dr. Hampson [35]. 


\section{Immunofluorescence localization studies}

HEK-293 cells stably transfected with a myc-tagged version of wild type mouse GPRC6A or the mutant in which KY was substituted for RKLP sequence in the 3rd intracellular loop were fixed by incubating the cells in $4 \%$ paraformaldehyde in phosphate buffered saline (PBS) for $10 \mathrm{~min}$ at room temperature. The cells were then permeabilized by incubation in PBS containing $0.05 \%$ saponin for $10 \mathrm{~min}$. The cells were then washed and incubated with a mouse anti-myc tag antibody (Cell Signalling) followed by donkey anti-mouse IgG conjugated to Alexa 488 (Molecular Probes). After washing the cells were imaged using a Zeiss AxioImager II microscope.

\section{Isolation of membrane fractionation}

The membrane protein was extracted from PC-3 cells using Mem-PER ${ }^{\mathrm{TM}}$ Plus Membrane Protein Extraction Kit (ThermoScientific; 89842), following the manufacturer's instruction. Total protein was extracted using Cell Extraction Buffer (ThermoScientific; FNN0011). $15 \mu \mathrm{g}$ of total or membrane protein was loaded for Western blot analysis.

\section{Site-directed mutagenesis}

The KY mutation in mGPRC6A was introduced using the QuikChange site-directed mutagenesis kit (Stratagene) with specific oligonucleotides, mGPRC6A.KYfor: 5'-GCATTCAAGGGCAAATATGAGAATTACAACGA AGCC-3' and mGPRC6A.KYrev: 5'-GGCTTCGTTGT AATTCTCATATTTGCCCTTGAATGC-3'. All final constructs were verified by sequencing on both strands.

\section{RT-PCR}

The primer set used to amplify this specific region of GPRC6A are: hGPRC6A.F2240, 5'-GAATGTCTCCTT GCCCAGAG-3' and hGPRC6A.R2734, 5'-TCTTTG CTTTTCTGCCAGGT-3'. The RT-PCR products were isolated and purified using QIAquick Gel Extraction kit (Qiagen) and sequenced using the hGPRC6A.F2240 primer.

\section{Targeting GPRC6A in PC-3 cells using CRISPR/Cas9}

The short guide RNA (sgRNA; sgRNA1 and 3) targeting exon 3 of the human GPRC6A gene (NM_001286354.1) were designed by Zhang Lab (http://crispr.mit.edu/), and synthesized to make the lenti-GPRC6A-sgRNA3-Cas9 constructs as described previously [36]. In brief, the 25bp DNA oligos containing the 20 bp target sequence and BsmbI sticky end were annealed and inserted into the lentiCRISPR-v2 plasmid (Addgene; 52961) digested with BsmbI (Fermentas). The DNA sequence for generating sgRNA1 and sgRNA3 are as follows: sgRNA1 forward: 5'- CACCgATCCCGAATGGCATAACCA-3' and
sgRNA1 reverse: 5'-AAACTGGTTATGCCATTCGGGATc-3', sgRNA3 forward: 5'-CACCgCCCCAACGCC TTTCAACCAT-3', sgRNA3 reverse: 5'-AAACATGGTT GAAAGGCGTTGGGGc-3'. For the control plasmid, no sgRNA sequence was inserted into the construct. GPRC6A edited cells and control cells were selected using $4 \mu \mathrm{g} / \mathrm{ml}$ puromycin. To determine the genomeediting effect, genomic DNA was extracted from the GPRC6A edited cells and control cells, and analyzed by sequencing PCR amplification products of the edited region. The PCR primers are as follows: hGPRC6A.Fmut: 5'-GCCCAGGTTAATGTCATTGT-3'; and hGPRC6A. Rmut: 5'-GGTAGGTCAGAGTAGGAAGTG-3'. The editing of GPRC6A was validated by western blot and Real-time PCR.

\section{Cell proliferation and migration assays}

For proliferation assays, $4 \times 10^{3}$ GPRC6A edited cells and control cell were seeded in the each well of 96-well plate, with or without various concentrations of osteocalcin $(10,50,100$, and $150 \mathrm{ng} / \mathrm{ml})$, and the MTT dye production method was used to measure for cell proliferation (Cayman Chemical, \# 10009365). A cell migration assay was performed using the CytoSelect 24-well cell migration Colorimetric Format assay (Cell Biolabs; CBA-100-C), following the manufacturer's instructions. In brief, each well contained a Boyden chamber with an $8-\mu \mathrm{m}$ pore polycarbonate membrane and media containing $10 \%$ FBS with or without $100 \mathrm{ng} / \mathrm{ml}$ osteocalcin. Chambers were seeded with $10^{5}$ GPRC6A edited cells or control cells expressing GPRC6A in serum free media and incubated at $37{ }^{\circ} \mathrm{C} 24 \mathrm{~h}$. Cells within wells were washed away, and migratory cells were stained and counted using a microplate reader at OD $570 \mathrm{~nm}$.

\section{Western blot}

Western blots were performed as previously described [37]. The antibodies used for these analyses were the following: GPRC6A antibody (ASSAY BIOTECHNOLOGY, \#G321); Phospho-p44/42MAPK (ERK1/2)(Thr202/Tyr204) antibody (Cell Signaling,\#9101); p44/42MAPK (ERK1/ 2) (Cell Signaling,\#9102); Phospho-mTOR (Ser2448) antibody (Cell Signaling,\#2971); Phospho-Akt (Ser473) antibody (\#9271).

\section{Real-time PCR}

All the primers used for this study are listed in Table 1. Samples were amplified for 40 cycles in an iCycler iQ real-time PCR detection system (Bio-Rad) with an initial melt at $95^{\circ} \mathrm{C}$ for $10 \mathrm{~min}$, followed by 40 cycles of $95^{\circ} \mathrm{C}$ for $15 \mathrm{~s}$ and $\mathrm{Tm}$ for $1 \mathrm{~min}$. PCR product accumulation was monitored at multiple points during each cycle by measuring the increase in fluorescence caused by the binding of SYBR Green I to double-stranded DNA. The 
Table 1 Primers for qRT-PCR

\begin{tabular}{|c|c|c|c|c|}
\hline Transcript & Primer sequences & Product length (bp) & Annealing temp $\left({ }^{\circ} \mathrm{C}\right)$ & Accession Number \\
\hline GPRC6A & $\begin{array}{l}\text { F:CCGGGACATATCATAATTGGG } \\
\text { R:CATTGCCACTGTGACTTCTGT }\end{array}$ & 234 & 58 & NM_148963 \\
\hline PCNA & $\begin{array}{l}\text { F:CCTGCTGGGATATTAGCTCCA } \\
\text { R:CAGCGGTAGGTGTCGAAGC }\end{array}$ & 109 & 61 & NM_002592 \\
\hline c-Fos & $\begin{array}{l}\text { F:GAGGGAGCTGACTGATACAC } \\
\text { R:CTCGGGGTAGGTGAAGACGA }\end{array}$ & 568 & 60 & NM_005252 \\
\hline MMP2 & $\begin{array}{l}\text { F:TACAGGATCATTGGCTACACACC } \\
\text { R:GGTCACATCGCTCCAGACT }\end{array}$ & 90 & 60 & NM_004530 \\
\hline MMP9 & $\begin{array}{l}\text { F:TGTACCGCTATGGTTACACTCG } \\
\text { R:GGCAGGGACAGTTGCTTCT }\end{array}$ & 97 & 58 & NM_004994 \\
\hline VEGF & $\begin{array}{l}\text { F:CAAGACAAGAAAATCCCTGTGG } \\
\text { R:CCTCGGCTTGTCACATCTG }\end{array}$ & 161 & 58 & M32977 \\
\hline BMP3 & $\begin{array}{l}\text { F:GCAGGGAGAGAGACCGAAG } \\
\text { R:TGGACCGTGCTGTACCTGT }\end{array}$ & 163 & 58 & NM_001201 \\
\hline PSA & $\begin{array}{l}\text { F:GTGTGTGGACCTCCATGTTATT } \\
\text { R:CCACTCACCTITCCCCTCAAG }\end{array}$ & 160 & 60 & AJ512346 \\
\hline RUNX2 & $\begin{array}{l}\text { F:TCAACGATCTGAGATTTGTGGG } \\
\text { R:GGGGAGGATTTGTGAAGACGG }\end{array}$ & 81 & 58 & NM_001015051 \\
\hline $\mathrm{OCN}$ & $\begin{array}{l}\text { F:GGCGCTACCTGTATCAATGG } \\
\text { R:GTGGTCAGCCAACTCGTCA }\end{array}$ & 110 & 60 & NM_199173 \\
\hline HSD17B11 & $\begin{array}{l}\text { F:CTGGCGAAGTGCGTGAGATT } \\
\text { R:GAGTACGCTITCCCAATTCCAT }\end{array}$ & 133 & 58 & NM_016245 \\
\hline HSD3B1 & $\begin{array}{l}\text { F:CACATGGCCCGCTCCATAC } \\
\text { R:GTGCCGCCGTITTCAGATTC }\end{array}$ & 90 & 58 & NM_000862 \\
\hline AKR1C3 & $\begin{array}{l}\text { F:TCTGGGATCTCAACGAGACAA } \\
\text { R:TGGAACTCAAAAACCTGCACG }\end{array}$ & 207 & 58 & NM_003739 \\
\hline Cyclophilin A & $\begin{array}{l}\text { F:CTGCACTGCCAAGACTGAGTG } \\
\text { R:CCACAATGTTCATGCCTTCT }\end{array}$ & 77 & 58 & NM_008907 \\
\hline
\end{tabular}

threshold cycle [38] of tested-gene product from each group was normalized to the $\mathrm{Ct}$ of cyclophilin $\mathrm{A}$, the $2^{-\Delta \Delta C t}$ method was employed to quantify and normalize the expression data.

\section{Xenograft model}

Athymic nude mice were purchased from Charles River laboratories. Mice were maintained and used in accordance with recommendations as described (National Research Council. 1985; Guide for the Care and Use of Laboratory Animals DHHS Publication NIH 86-23, Institute on Laboratory Animal Resources, Rockville, MD) and following guidelines established by the University of Tennessee Health Science Center Institutional Animal Care and Use Committee. The animal study protocol was approved by the institutional review board at University of Tennessee Health Science Center Institutional Animal Care and Use Committee.

GPRC6A edited PC-3 cells or control cells were labeled with a lentiviral vector expressing luciferase (pLenti-UBC-Luc2-T2A-mKate) and $2 \times 10^{6}$ cells were mixed with Matrigel (Corning, \#356234) and subcutaneously injected into the left and right flank of 5-week- old nude mice, and the mice were administrated $3 \mathrm{ng} / \mathrm{g}$ osteocalcin or vehicle per day by IP injection. Tumor growth was monitored over a 28-day period using a Xenogen imaging system (PerkinElmer). Mice were then sacrificed and tumors were dissected free of connective tissue before analysis. Immunohistochemistry (IHC) analysis was accomplished by Vectastain ABC kit (Vector Laboratories, PK-6200), and the antibodies used for IHC were obtained from commercial sources and diluted for use as follows: GPRC6A (1:100; ASSAY BIOTECHNOLOGY, \# G321); and RUNX2 (1:100, Abcam, \#b23981); PCNA (1:100, Invitrogen, \#133900). Staining intensity was performed by assessing Vector NovaRed color (Vector Laboratories, SK-4800) in $3 \mathrm{sec}-$ tions of tumor for each antibody.

\section{Statistical analysis of data}

We evaluated differences between groups by one-way analysis of variance, followed by a post-hoc Tukey's test. Significance was set at $p<0.05$. All values are expressed as means \pm SEM. All computations were performed using the Statgraphic statistical graphics system (STSC Inc.). 


\section{Results}

Human GPRC6A transcripts are expressed in prostate cancer cells

We confirmed that GPRC6A message is present in several human prostate cancer cell lines [12], including androgen insensitive PC-3 cells (derived from lumbar metastasis), DU145 cells (derived from CNS metastasis), and 22Rv1 cells (derived from androgen insensitive xenograft), and androgen sensitive LNCap cells (derived from lymph node metastasis) (Fig. 1a). The levels of GPRC6A transcripts were highest in PC-3 and DU145 cell lines.
Because of questions regarding the cell surface expression of GPRC6A [28, 29], and the importance of the 3rd intracellular loop (3rd IL) in GPCR membrane trafficking [39], we sequenced this region in DU145, PC-3, LNCap and 22Rv1 human prostate cancer cell lines [28]. The cDNA sequence encoding the 3rd IL from all four human prostate cancer cells had the AAATAT nucleotide sequence encoding the "K..Y" amino acid sequence [the human reference at amino acid 774 (Lys774Tyr775)] shown in Fig. 1b. The human GPRC6A 774-KY-775 motif in the 3rd IL represents an insertion/deletion that replaced the ancestral 774-

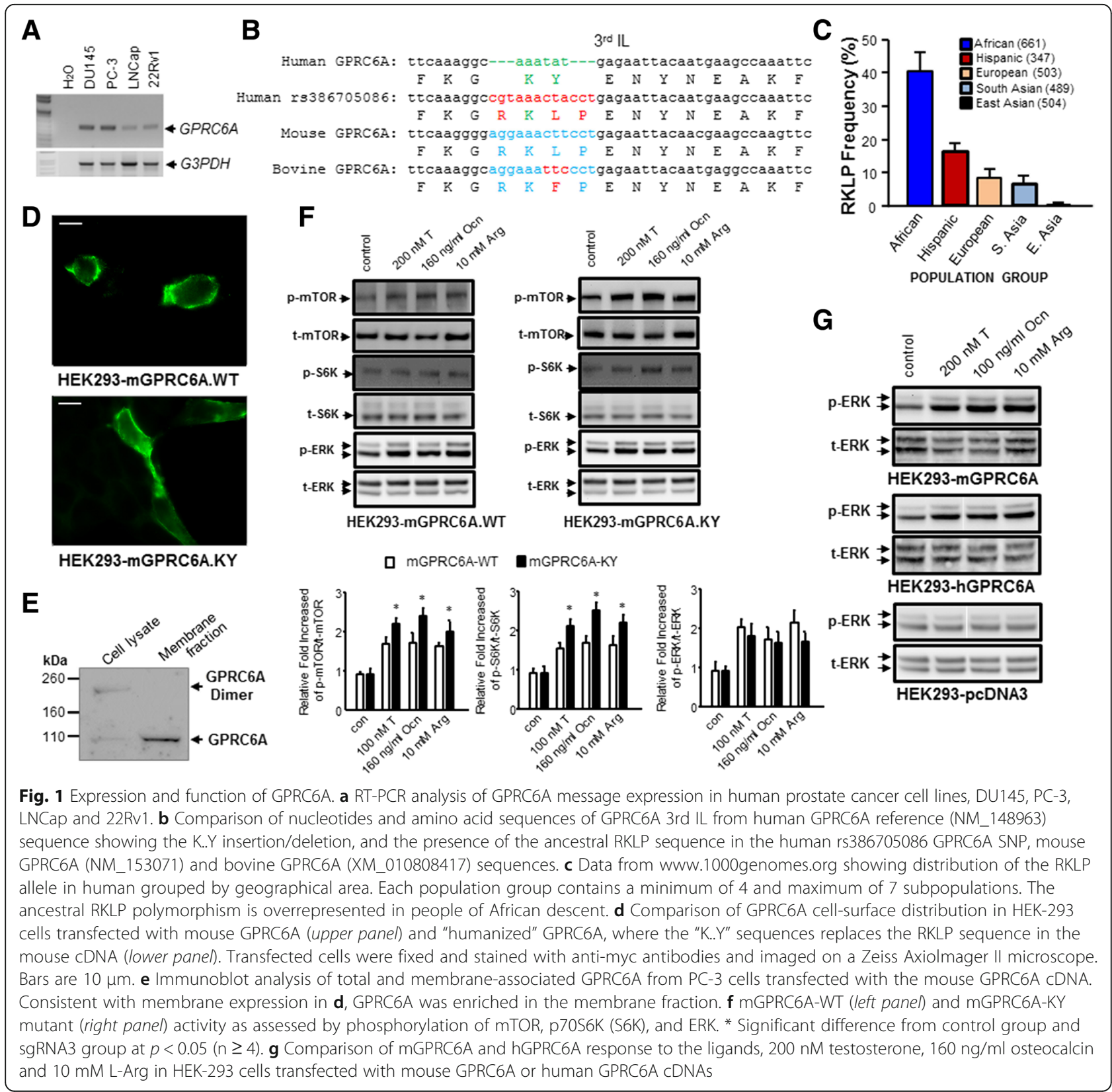


RKLP-777 sequence [variation rs386705086 (p.Lys_ Tyr775 replace to ArgLysLeuPro); except for 775RKFP-778 in the bovine sequence] (Fig. 1b). The "K..Y" deletion/insertion emergenced only in hominids, and is found in greater than $84 \%$ of humans [38, 40]. The ancestral "RKLP" sequence present in all other animal species is considered to be a polymorphism (rs386705086) in humans (Fig. 1b), and is retained with an allele frequency $0.6 \%$ in people of East Asian descent, $6.6 \%$ of South Asians, $8.2 \%$ of Europeans, $16.6 \%$ of Hispanics, and $40.1 \%$ in people of African descent (Fig. 1c, Data from www.1000genomes.org). The emergence of the "K..Y" polymorphism only in hominids and its preservation during evolution suggests a selection pressure that modified the functions of this allele in the majority of humans [33].

\section{Cell distribution and ligand activation of GPRC6A}

Next, we compared the cell distribution and function of a wild-type mouse GPRC6A cDNA constructs (mGPRC6A.WT) and a mutated mouse GPRC6A construct in which the "RKLP" sequence was replaced with the human "K..Y" sequence (mGPRC6A.KY). Results from transfection of HEK-293 cells with the mGPRC6A.WT and mGPRC6A.KY are shown in Fig. 1d. We observed no significant differences in the cellular distribution of mouse GPRC6A containing the RKLP sequence and the mouse GPRC6A cDNA containing the K..Y sequence in 3rd IL. Both wild-type and "humanized" mouse GPRC6A were predominantly expressed on the surface of cells (Fig. 1d, upper and bottom panel, respectively). We also have compared the protein levels of GPRC6A from a total cell lysate and membrane fraction prepared from PC-3 cells (Fig. 1e). The western blot indicates that GPRC6A is enriched in the membrane fraction prepared from PC-3 cells (Fig. 1e).

To determine if the K..Y polymorphism affects liganddependent activation of GPRC6A in these cells, we tested the ability of the GPRC6A ligands, L-Arg, osteocalcin and testosterone, to activate ERK-signaling. Transfection of the wild-type or "humanized" mouse GPRC6A into HEK-293 cells resulted in similar ERK signaling in response to ligand stimulation of GPRC6A (Fig. 1f). HEK-293 cells transfected with the human or mouse wildtype GPRC6A cDNAs also equally increased ERK activity in response to $10 \mathrm{mM} \mathrm{L}$-Arg, $160 \mathrm{ng} / \mathrm{ml}$ osteocalcin, or $200 \mathrm{nM}$ testosterone, respectively (Fig. 1g). However, the K..Y mutant preferentially activated mTOR and p70S6K phosphorylation in the mTOR pathway compared to the wild-type RKLP mouse GPRC6A (Fig. 1f).

\section{Editing GPRC6A in PC-3 cells using CRISPR/Cas9}

To further investigate the function of endogenous human GPRC6A, we used CRISPR/Cas9 editing to mutate the GPRC6A gene in the PC-3 s cells. We used a lentiCRISPR vector (Fig. 2a) and designed sgRNAs (sgRNA1 and 3) against exon 3 of GPRC6A (Fig. 2b). We designed two sgRNAs against exon 3 to avoid non-specific effects of the CRISPR/Cas9 system. Genomic DNA PCR products were cloned into a plasmid and the GPRC6A gene targeted by the sgRNAs was sequenced to analyze the targeted GPRC6A genomic region in the transfected PC-3 cells. This analysis revealed that the sequence of the GPRC6A gene was specifically altered in $\sim 80 \%$ of the cells that would disrupt gene function, including insertions or deletions at the sites targeted by sgRNA1 and 3 (Additional file 1: Figure S1). Western blotting (Fig. 2c) and real-time PCR (Fig. 2d) confirmed that GPRC6A protein and mRNA expression were significantly decreased in PC-3 cells edited using sgRNA-1 and sgRNA3. Furthermore, editing PC cells with sgRNA3 resulted in a greater decrease in GPRC6a protein and mRNA expression than that observed with sgRNA1s (Fig. 2c and d).

\section{CRISPR/Cas9 editing of GPRC6A in PC-3 cells attenuates ligand dependent prostate cancer responses in vitro}

In prior studies we show that ligand-mediated activation of GPRC6A is coupled to ERK and AKT phosphorylation $[37,41]$. Therefore, we assessed ERK activation in response to GPRC6A ligands in PC-3-sgRNA1, PC-3-sgRNA3, and PC-3 control cells. We found that $20 \mathrm{mM} \mathrm{L-Arg,} 100 \mathrm{ng} /$ $\mathrm{ml}$ osteocalcin and $100 \mathrm{nM}$ testosterone stimulated phospho-ERK activity in control PC-3 cells (Fig. 3a, upper panel), and this response was significantly decreased in PC-3 cells edited using sgRNA1 and sgRNA3 guide RNAs (Fig. 3a). In PC-3-sgRNA3 cells there was a significant reduction in ERK activity stimulated by L-Arg, osteocalcin and testosterone (Fig. 3a, lower panel), whereas PC-3sgRNA1 cells showed a partial response to L-Arg and osteocalcin, but no response to testosterone at the concentration tested (Fig. 3a, lower panel).

To test the dose-dependent effects of ligand activation of GPRC6A, we examined the effects of osteocalcin at doses of $0,10,50,100,150 \mathrm{ng} / \mathrm{ml}$ on ERK and Akt phosphorylation in control and PC-3-sgRNA3 cells. We observed that Osteocalcin stimulated ERK (Fig. 3b) and AKT (Fig. 3c) phosphorylation in a dose-dependent manner in control PC-3 cells transfected with empty lentiCRISPR vector. Osteocalcin stimulated ERK and AKT phosphorylation were significantly attenuated in PC-3-sgRNA3 cells (Fig. $3 \mathrm{~b}$ and c). Osteocalcin also stimulated the phosphorylation of mTOR, a downstream effector of ERK and AKT, in control PC-3 cells, but mTOR phosphorylation was significantly reduced in osteocalcin stimulated PC-3-sgRNA3 cells (Fig. 3d).

We assessed the effects of GPRC6A on prostate cancer growth and cell migration. The reduced expression of GPRC6A in PC-3-sgRNA3 cells had a small but significant effect on osteocalcin-stimulated increase in PC-3 cell 

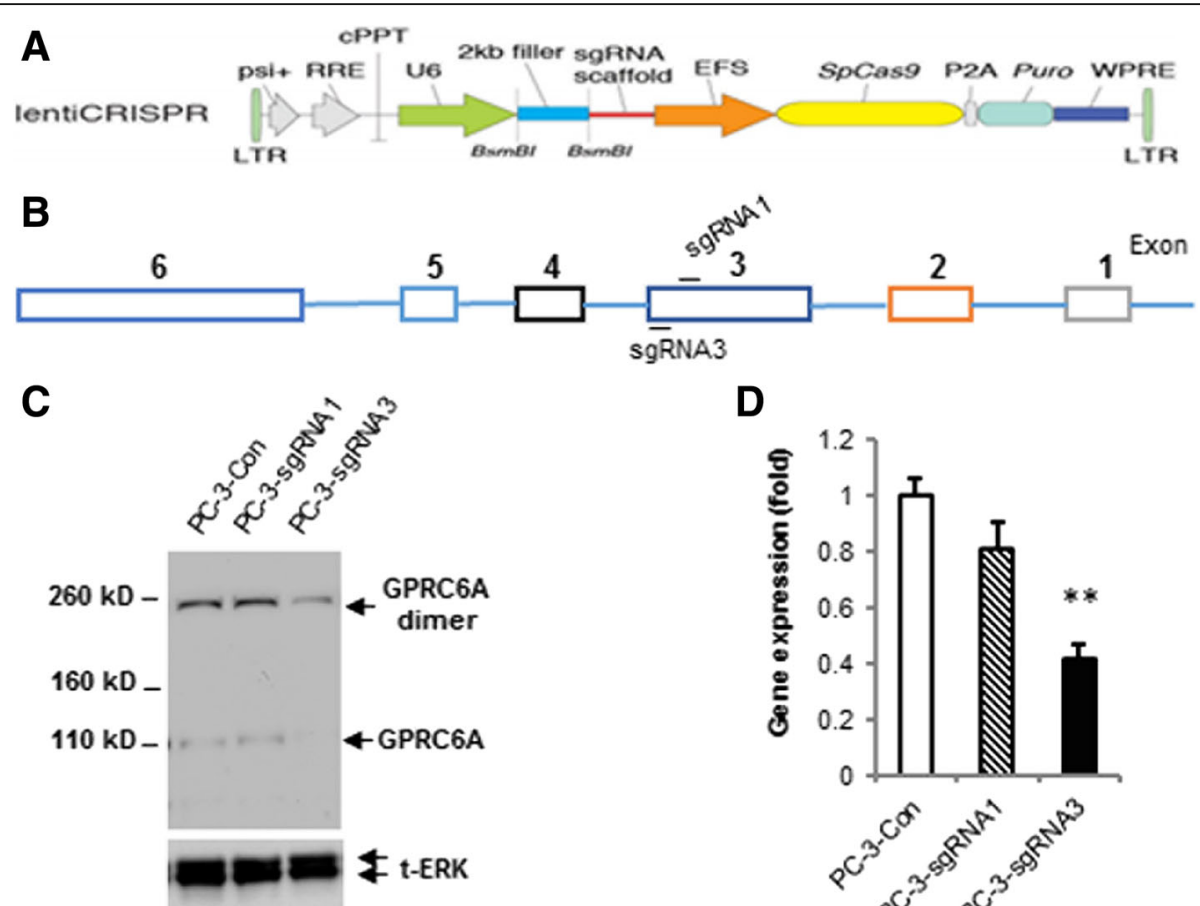

D

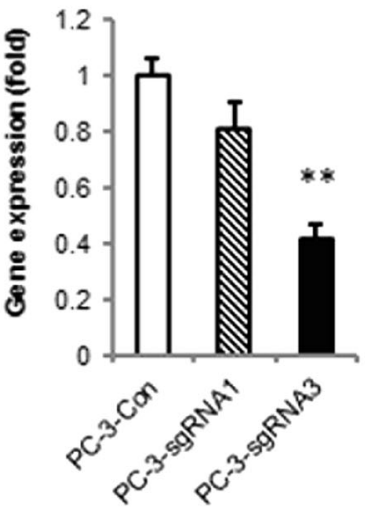

Fig. 2 Editing GPRC6A in PC-3 cells using CRISPR/Cas9. Strategy of targeting GPRC6A in human prostate cancer PC-3 cells using CRISPR/Cas9 and the vector of lentiCRISPR v2 (the figure is adopted from http://genome-engineering.org/gecko/wp-content/uploads/2013/12/lentiCRISPRv2-and-lentiGuideoligo-cloning-protocol.pdf) (a) and GPRC6A gene structure (b). Blue bars show sgRNA1 and sgRNA3 location in exon 3 of GPRC6A gene. Short guide RNAs (sgRNA) designed to target exon 3 of GPRC6A gene. The controls were empty vector, with no inserts in this region. CRISPR/Cas9 editing in PC-3 human prostate cancer cells decreased GPRC6A protein by Western blot (c) and mRNA by real-time PCR (d). ** Significant difference from control group and sgRNA3 group at $p<0.01(n \geq 4)$

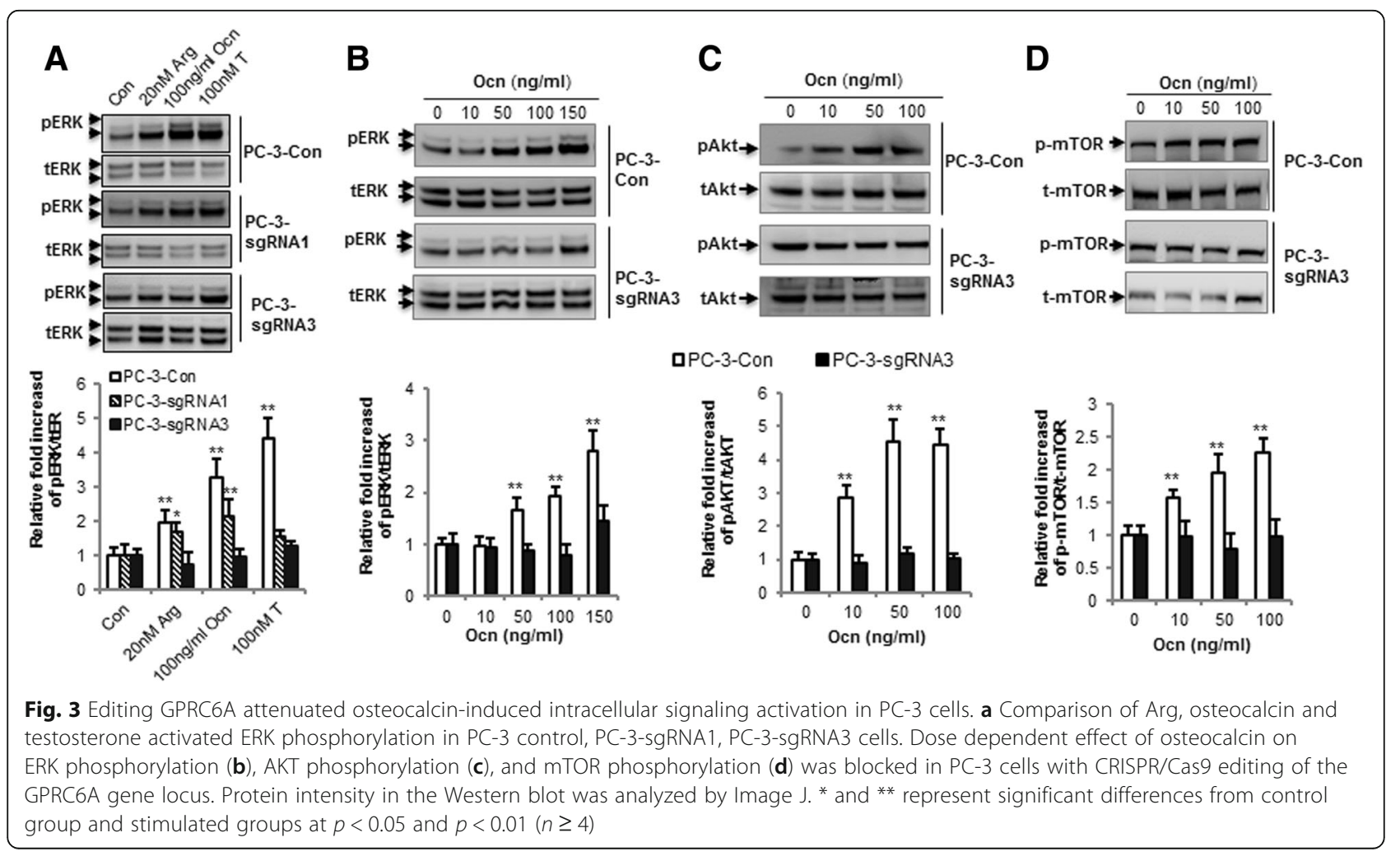


number (Fig. 4a). GPRC6A gene editing of exon 3 using CRISPR/Cas9 significantly decreased osteocalcin-induced PCNA expression, a cell proliferation marker gene (Fig. 4b). Cell migration was also significantly decreased in PC-3-sgRNA3 cells compared to PC-3 controls in the absence of osteocalcin; and the addition of osteocalcin to the culture media resulted in a significant $72 \%$ increase in control PC-3 cell migration, and this response was completely inhibited in PC-3-sgRNA3 cells (Fig. 4c).

We also examined by real-time PCR the effects of ligand stimulated GPRC6A on a panel of genes known to be associated with prostate cancer progression. We found that the mRNA levels of $c$-Fos and the migration-related genes, MMP9, VEGF and BMP3, were significantly increased by osteocalcin stimulation of PC-3 control cells, but not in PC-3-sgRNA3 cells (Fig. 5a-d). Prostate specific antigen [42] and Runt-related transcription factor 2 (RUNX2), a

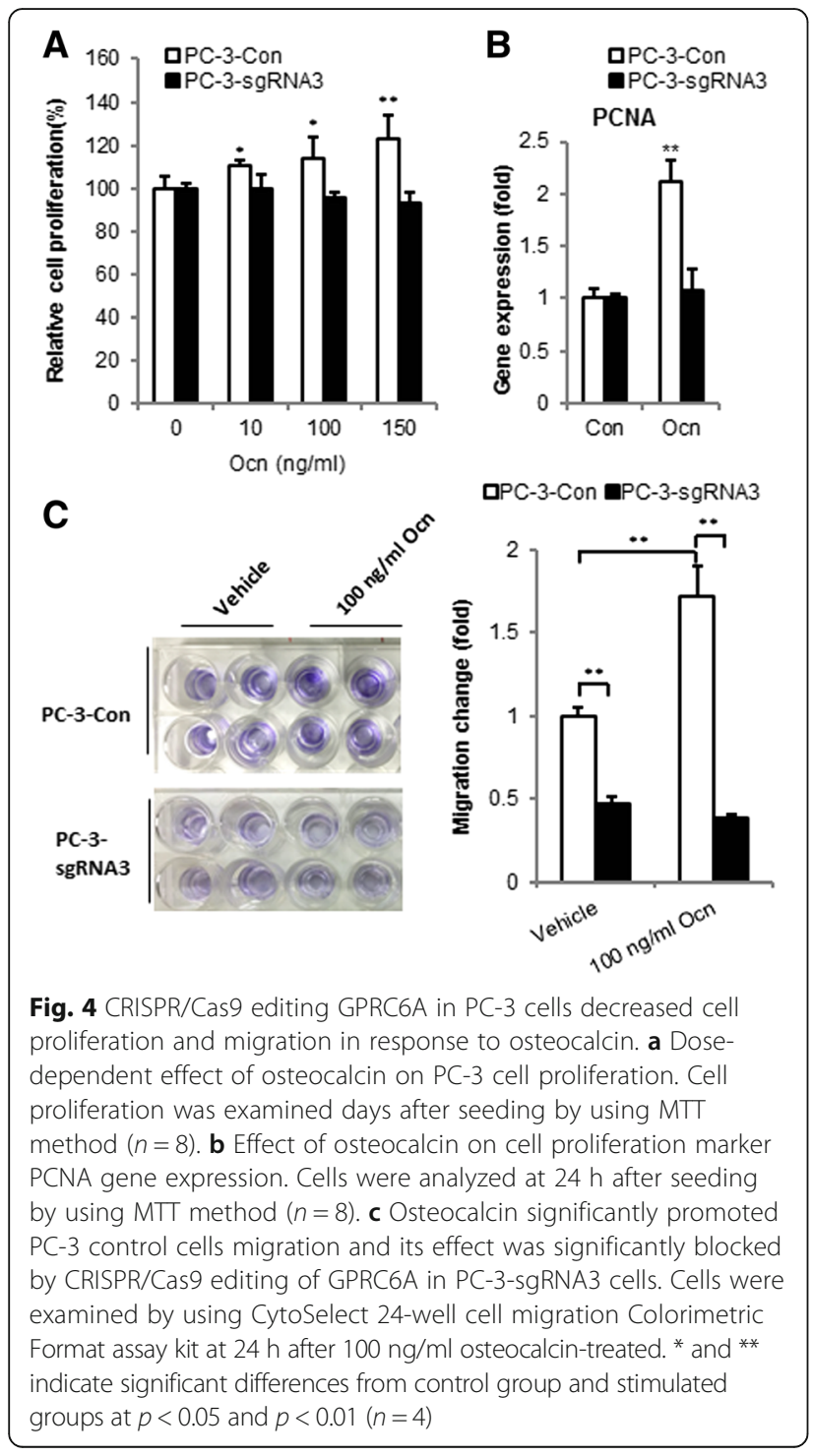

bone-specific transcriptional regulator expressed in metastatic prostate cancer cells, are regulated by ligand activation of GPRC6A [37]. We observed that osteocalcin significantly stimulated PSA, RUNX2 and osteocalcin $(O C N)$ message levels in PC-3 control cells, but not in PC-3-sgRNA3 cells (Fig. 5e-g). We also tested the effects of GPRC6A activation on testosterone biosynthesis in prostate cancer cells [15]. Osteocalcin addition to PC-3 control cells significantly increased HSD17b11, HSD3B1, and $A K R 1 C 3$, which are involved in testosterone biosynthesis. In contrast, osteocalcin did not increase the expression of these genes in PC-3-sgRNA3 cells (Fig. $5 \mathrm{~h}-\mathrm{j}$ ).

\section{CRISPR/Cas9 editing of GPRC6A in PC-3 cells decreases prostate cancer tumor growth in vivo}

Finally, to investigate the effects of GPRC6A on regulation of prostate cancer cell progression in vivo, we performed human xenograft studies in nude mice. Human PC-3 control cells and PC-3-sgRNA3 cells expressing a luciferase reporter were injected subcutaneously (s.c.) into the lower flank of 5 week-old nude mice followed by treatment with either vehicle or osteocalcin at a daily dose of $3 \mathrm{ng} / \mathrm{g}$ by IP injection. Tumor growth was assessed by luciferase intensity (Fig. 6a) and mass was assessed by weighing dissected tumors (Fig. 6b). In vehicle treated mice, implanted control PC-3 cells exhibited significant increases in tumor growth (Fig. 6a) and weight (Fig. 6b) compared to mice implanted with GPRC6A PC-3-sgRNA3 cells. Osteocalcin treatment significantly increased tumor growth and weight in PC3 control xenografts (Fig. 6a and b). In contrast, osteocalcin administration resulted in an attenuated, but non-significant increase in tumor growth and no change in tumor gross size or weight in mice implanted with PC-3-sgRNA3 cells (Fig. 6a and b). Immunohistochemical staining revealed a dramatic reduction in the protein expression of GPRC6A in the tumors of PC-3sgRNA3 cells xenografts compared to PC-3 control cells (Fig. 5c, upper panel). Osteocalcin-stimulation did not alter the expression of GPRC6A (Fig. 5c, upper panel). We observed a significant increase in PCNA and RUNX2 protein levels in tumors derived from PC3 control cells treated with osteocalcin ( $3 \mathrm{ng} / \mathrm{g}$ Osteocalcin, IP daily for 5 weeks); in contrast, treatment of PC-3-sgRNA3 xenografts with osteocalcin did not result in increased levels of PCNA and RUNX2 (Fig. 6c, middle and lower panels, respectively).

\section{Discussion}

In this study we tested if human GPRC6A has important tumor promoting effects in prostate cancer. Using a panel of human prostate cancer cell lines, we confirmed that human GPRC6A transcripts are expressed in all prostate cancer cell lines tested. Because cell surface 


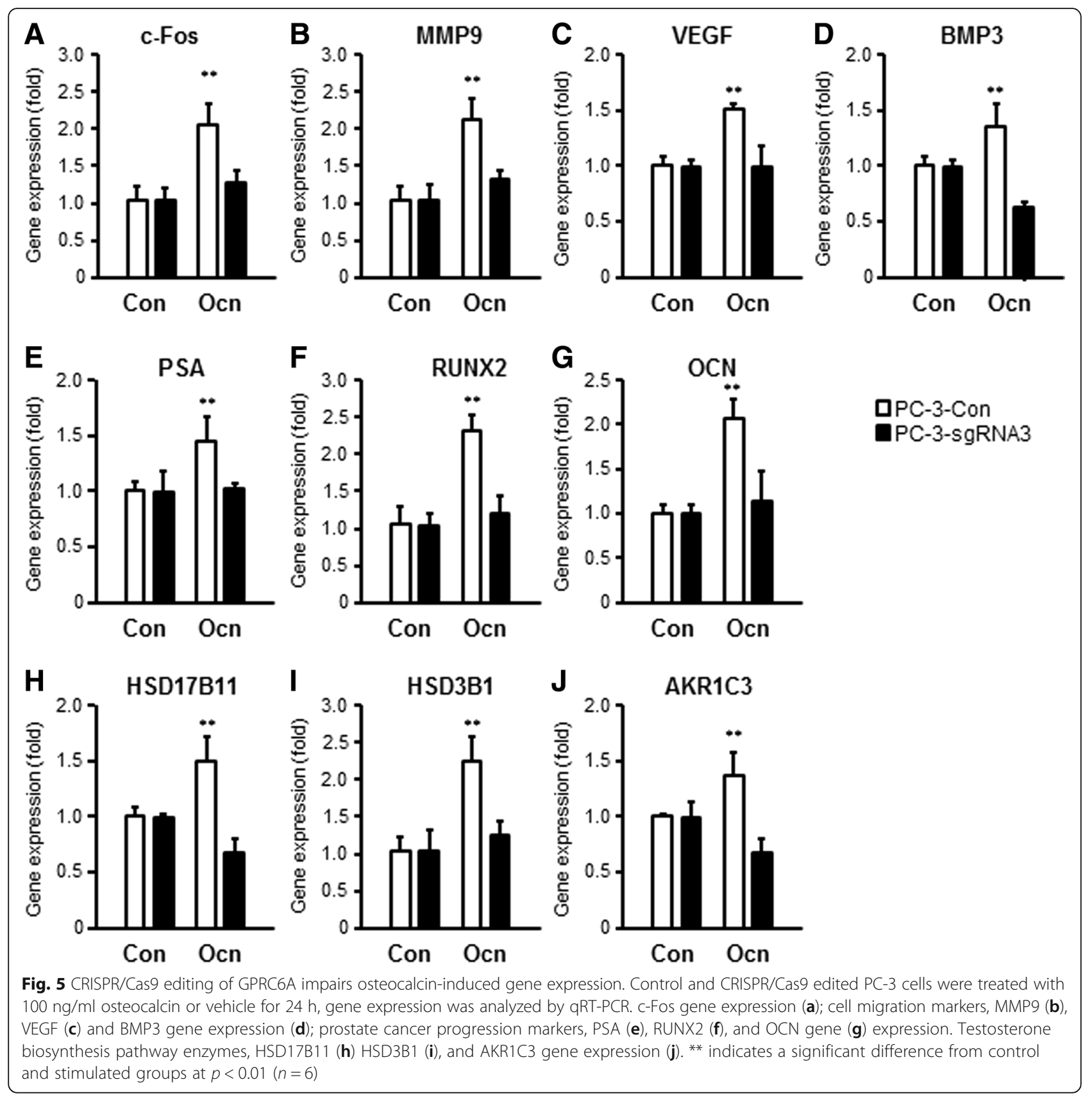

membrane localization of human GPRC6A has been questioned [28, 29, 33, 43], we sequenced the 3rd intracellular loop, a region of GPCRs that binds $\beta$-arrestins and regulates membrane trafficking $[28,29]$. We identified the presence of only the "K..Y" sequence in the 3rd IL in human GPRC6A. This sequence is noteworthy, because it evolved only in hominids and replaces the ancestral "RKLP" sequence present in all other species [40]. Comparision of the cellular localization of GPRC6A in HEK-293 cells transfected with wild-type mouse and a humanized mouse GPRC6A cDNA with the "K..Y" sequence showed that both localized to the cell surface. Human
GPRC6A containing the K..Y sequence (human reference sequence) has been shown by others to localize to the cell curface when expressed in HEK-239 T cells [15].

The cell surface localization of the 'humanized' mouse GPRC6A, however, differs from other studies of the human GPRC6A that show a punctate, intracellular-like distribution of this receptor in transfected HEK-293 cells $[33,44]$. Lv et al. [43] found that human GPRC6A exhibited approximately $30 \%$ cell surface expression, whereas the closely related calcium sensing receptor, CasR, which has the RKLP sequence in its 3rd IL, exhibited $>90 \%$ cell surface expression. Our data also, differ from the 


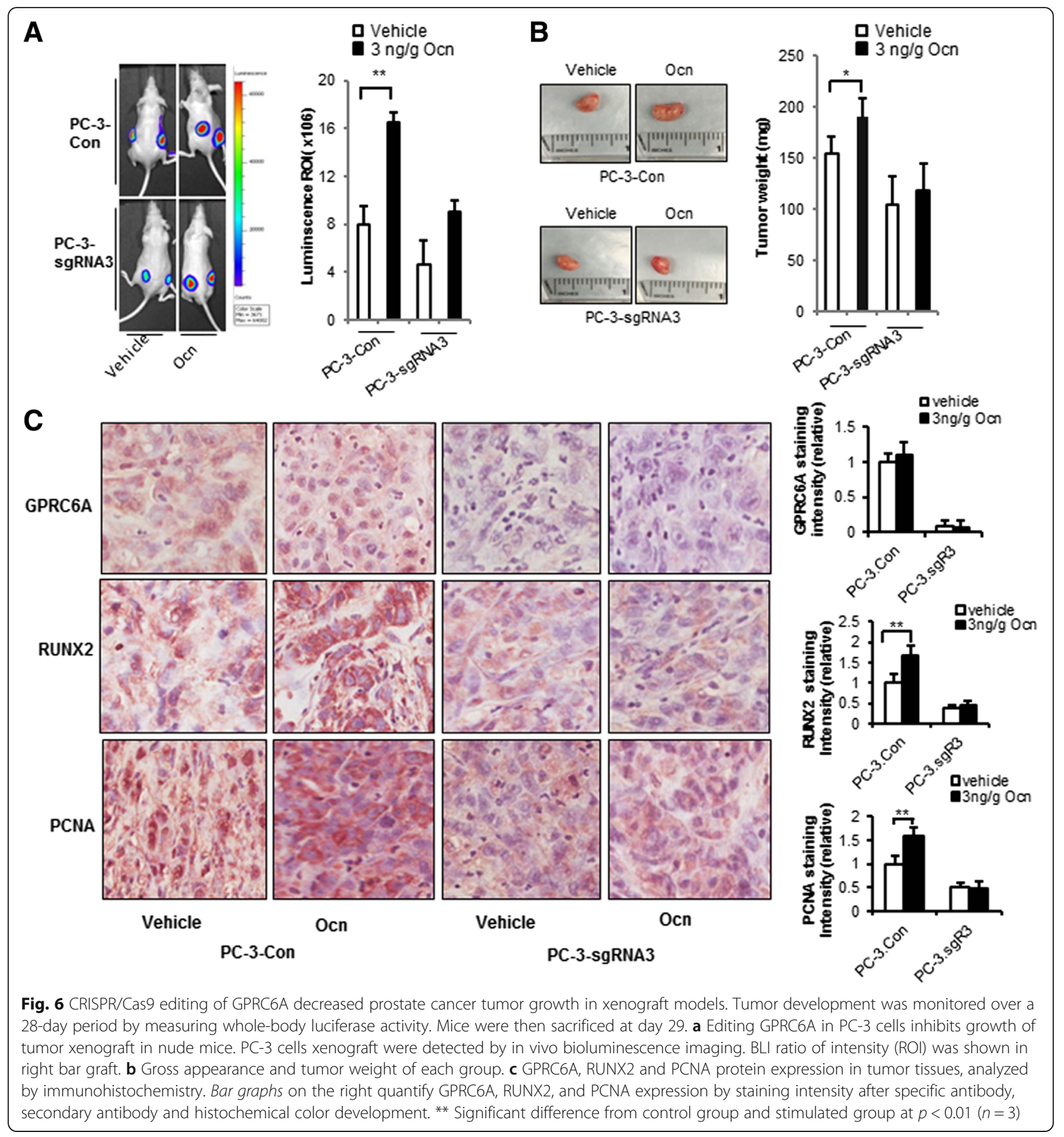

analysis of human/mouse chimeric GPRC6A constructs that replaced the entire 3rd IL, and concluded the respective K..Y and the RKLP inserts in human and mice determined the intracellular and cell surface membrane expression of these receptors. Replacing the RKLP sequence, however, with K..Y in the mouse receptor is not sufficient for intracellular retention of the mouse GPRC6A and that additional sequences in the 3rd IL or other domains likely regulate cell surface expression of GPRC6A [15, 33, 44].

Nevertheless, in heterologous cell systems transfected with different mouse and human GPRC6A constructs, we found that ligands activated GPRC6A encoded by both mouse and human constructs, irrespective of whether they contained the "K..Y" or "RKLP" polymorphisms. Indeed, transfection in HEK-293 cells of cDNAs encoding the 
mouse GPRC6A with the "RKLP" sequence, the human GPRC6A with the "K..Y" sequence, and a "humanized" mouse GPRC6A created by replacing the RKLP with "K..Y" sequence, all imparted the ability of ligands to stimulate ERK, Akt and mTOR phosphorylation.

Osteocalcin activation of GPRC6A has previously been shown to stimulate mTOR phosphorylation in myocytes and fibroblasts $[10,41]$. mTOR can be activated by cell surface receptors through PI3K/Akt or through an evolutionarily conserved intracellular amino acid lysosomal mTORC1 signaling pathway involving RAG GTPases, Ragulator, and vaculoar $\mathrm{H}^{+}$-ATPase [45]. Interestingly, the presence of the "K..Y" polymorphism resulted in a greater mTOR response, suggesting that the "K..Y" polymorphism may alter signaling through mTOR. There is a precident for $\beta$-arrestin-dependent regulation of endosomal signaling of the PTH GPCR [39], and it is tempting to speculate that the "K..Y" polymorphism in human GPRC6A may have evolved to alter GPRC6A's interactions with $\beta$-arrestin [16], and the kinetics of receptor recycling to allow for participation in this intracellular amino acid sensing pathway [45]. Further studies are needed to test this possibility.

More importantly, we established that endogenous human GPRC6A functionally responds in both in vitro and in vivo model systems. We used CRISPR/Cas9 to delete endogenous GPRC6A in androgen insensitive human PC-3 cells. GPRC6A edited PC-3 cells exhibited an attenuated response to L-Arg, osteocalcin and testosterone stimulation of ERK, Akt, and mTOR phosphorylation compared to controls, pathways involved in advanced prostate cancer and emergence of hormone-refractory disease [46]. In addition, activation of GPRC6A stimulated prostate cancer cell line proliferation and migration, whereas editing GPRC6A in PC-3 cells reduced cell proliferation and transcription of PCNA and c-Fos. CRISPR/Cas9 editing of PC-3 cells showed impaired ligand-stimulated expression of $M M P 9, V E G F, B M P 3$, $P S A, R U N X 2$, and $O C N$, which are involved in prostate cancer progression [2, 20, 47, 48]. In addition, GPRC6A edited PC-3 cells exhibited reduced ligand stimulated expression of transcripts encoding key enzymes regulating intra-tumor androgen biosynthesis, including 17-betahydroxysteroid dehydrogenase 11 (HSD17B11), hydroxydelta-5-steroid dehydrogenase, 3 beta- and steroid deltaisomerase 1 (HSD3B1), and aldo-keto reductase family 1 , member C3 (ARK1C3). Since PC-3 cells are androgenindependent with no or weak androgen receptor (AR) activity $[49,50]$, GPRC6A may respond to intra-prostatic androgen synthesis and contribute to the high metastatic potential of PC-3 cells. Osteocalcin activation of GPRC6A may indirectly promote androgen synthesis through stimulation of IL-6 [10], a cytokine that can promote androgen synthesis in prostate cancer cells through enhancing AKR1C3 transcription [51]. Overall, these findings are consistent with prior in vitro studies showing that activation of GPRC6A in human PC-3 and 22Rv1 cells results in ERK phosphorylation, cell proliferation, and chemotaxis [12]; that knockdown of GPRC6A by siRNA inhibited PC-3 prostate cancer cell migration and invasion, and that overexpression of GPRC6A promoted prostate cancer epithelial-mesenchymal transition [20].

Finally, the mouse PC-3 xenografts showed that activation of GPRC6A with osteocalcin enhances primary tumor growth, and that CRISPR/Cas9 induced mutations in GPRC6A in PC-3 cells resulted in reduced primary tumor growth and the further enhancement of tumor growth in response to osteocalcin administration. PCNA and RUNX2 expression was decreased and the ability of osteocalcin to increase PCNA and RUNX2 expression was attenuated in xenografts derived from PC-3 cells in which GPRC6a levels were reduced. These data, along with the observations that GPRC6A is upregulated in human prostate cell lines [12], and GWAS studies that have identified other polymorphisms in the GPRC6A gene associated with prostate cancer progression in men of Asian descent [21-23], support the notion that GPRC6A has biological functions in prostate cancer.

\section{Conclusion}

Available evidence suggests that GPRC6A and its ligands modulate the progression of prostate cancer. We previously demonstrated that GPRC6A can directly promote prostate cancer cell proliferation, migration, and in vivo tumor growth [12]. The current study shows that by activating GPRC6A, signaling via ERK, AKT, and mTOR is increased in prostate cancer cells. In addition, activation of GPRC6A can stimulate androgen biosynthesis pathways to regulate intra-tumoral steroidogenesis in prostate cancer cells [52], and in testicular Leydig cells to stimulate circulating testosterone concentrations. Our findings raise the possibility that the presence of osteocalcin in the bone micro-environment potentially creates paracrine signaling pathways that might explain the propensity of CRPC to metastasize to bone [53]; and expression of osteocalcin in malignant prostate cell lines and prostate cancer specimens potentially leads to autocrine activation of GPRC6A [54]. Our findings also suggest that GPRC6A, by mediating the non-genomic effects of testosterone [55], may provide alternative pathways to androgen receptor signaling in prostate cancer [26]. Finally, the persistence of the ancestral RKLP sequence and its predominant expression in humans of African descent suggest that this human polymorphism may contribute to the racial disparities in diseases associated with GPRC6A, including Metabolic Syndrome [56] and prostate cancer [57]. As such, GPRC6A is "a target of interest" for treating prostate cancer and warrants further efforts to develop antagonists for this receptor as potential treatments for prostate cancer. 


\section{Additional file}

Additional file 1: Figure S1. Targeting GPRC6A gene in PC-3 cells using CRIPR/Cas9 system. (DOCX 89 kb)

\section{Abbreviations}

Cas9: CRISPR-associated protein 9 nuclease; CRISPR: Clustered regularly interspaced short palindromic repeats; ERK: Extracellular signal-regulated kinases; GPRC6A: G Protein-Coupled Receptor Class C Group 6 Member A; K..Y: The human reference at amino acid 774 (Lys774Tyr775); MetS: Metabolic syndrome; RKLP: Variation rs386705086 (p.Lys_Tyr775 replace to ArgLysLeuPro); sgRNA: Short guide RNA

\section{Acknowledgements}

We thank Dr. Hampson from University of Ontario (Toronto, Canada) for providing mouse GPRC6A cDNA construct.

\section{Funding}

This work was supported by grants from the National Institutes of Health (R01-DK095812, R01-AR37308 and R01-DK092176), grant from American Diabetes Association (ADA-1-13-149-BR).

\section{Availability of data and materials}

None.

\section{Authors' contributions}

RY, MP and JC performed experiments. SKN provided important reagents and reviewed the manuscript. RY, MP, and LDQ designed the study and wrote and reviewed the manuscript. MP and LDQ are guarantors of this work and, as such, had full access to the data in the study and take responsibility for the integrity of the data and the accuracy of the data analysis. All authors read and approved the final manuscript.

\section{Ethics approval and consent to participate}

The procedures of this study (15-150) were approved by the Institutional Review Board of University of Tennessee Health Science Center Institutional Animal Care and Use Committee.

\section{Consent for publication}

Not applicable.

\section{Competing interests}

The authors declare that they have no competing interests.

\section{Publisher's Note}

Springer Nature remains neutral with regard to jurisdictional claims in published maps and institutional affiliations.

\section{Author details}

${ }^{1}$ Department of Medicine, University of Tennessee Health Science Center, 19 S Manassas St., Memphis, TN 38163, USA. Department of Microbiology, Immunology and Biochemistry, University of Tennessee Health Science Center, 19 S Manassas St., Memphis, TN 38163, USA.

Received: 21 February 2017 Accepted: 20 June 2017

Published online: 28 June 2017

\section{References}

1. Siegel RL, Miller KD, Jemal A. Cancer statistics, 2016. CA Cancer J Clin. 2016;66:7-30

2. Huang W-C, Xie Z, Konaka H, Sodek J, Zhau HE, Chung LW. Human osteocalcin and bone sialoprotein mediating osteomimicry of prostate cancer cells: role of cAMP-dependent protein kinase A signaling pathway. Cancer Res. 2005:65:2303-13.

3. Pi M, Wu Y, Quarles LD. GPRC6A mediates responses to osteocalcin in $\beta$-cells in vitro and pancreas in vivo. J Bone Miner Res. 2011;26:1680-3.

4. Pi M, Chen L, Huang M-Z, Zhu W, Ringhofer B, Luo J, Christenson L, Li B, Zhang J, Jackson PD. GPRC6A null mice exhibit osteopenia, feminization and metabolic syndrome. PLoS One. 2008;3:e3858.
5. Pi M, Kapoor K, Ye R, Nishimoto SK, Smith JC, Baudry J, Quarles LD. Evidence for osteocalcin binding and activation of GPRC6A in $\beta$-cells. Endocrinology. 2016;157:1866-80

6. Oury F, Sumara G, Sumara O, Ferron M, Chang H, Smith CE, Hermo L, Suarez S, Roth BL, Ducy P. Endocrine regulation of male fertility by the skeleton. Cell. 2011;144:796-809.

7. Mizokami A, Yasutake Y, Higashi S, Kawakubo-Yasukochi T, Chishaki S, Takahashi I, Takeuchi H, Hirata M. Oral administration of osteocalcin improves glucose utilization by stimulating glucagon-like peptide-1 secretion. Bone. 2014;69:68-79.

8. Oya M, Kitaguchi T, Pais R, Reimann F, Gribble F, Tsuboi T. The G proteincoupled receptor family $C$ group 6 subtype A (GPRC6A) receptor is involved in amino acid-induced glucagon-like peptide-1 secretion from GLUTag cells. J Biol Chem. 2013;288:4513-21.

9. Otani T, Mizokami A, Hayashi Y, Gao J, Mori Y, Nakamura S, Takeuchi H, Hirata M. Signaling pathway for adiponectin expression in adipocytes by osteocalcin. Cell Signal. 2015;27:532-44.

10. Mera P, Laue K, Ferron M, Confavreux C, Wei J, Galán-Díez M, Lacampagne A Mitchell SJ, Mattison JA, Chen Y. Osteocalcin signaling in myofibers is necessary and sufficient for optimum adaptation to exercise. Cell Metab. 2016;23:1078-92.

11. Mera P, Laue K, Wei J, Berger JM, Karsenty G. Osteocalcin is necessary and sufficient to maintain muscle mass in older mice. Mol Metabol. 2016:5:1042-7.

12. Pi M, Quarles LD. GPRC6A regulates prostate cancer progression. Prostate. 2012;72:399-409.

13. Lee NK, Sowa H, Hinoi E, Ferron M, Ahn JD, Confavreux C, Dacquin R, Mee PJ McKee MD, Jung DY. Endocrine regulation of energy metabolism by the skeleton. Cell. 2007;130:456-69.

14. Bhindi B, Xie WY, Kulkarni GS, Hamilton RJ, Nesbitt M, Finelli A, Zlotta AR, Evans A, van der Kwast TH, Alibhai SM. Influence of metabolic syndrome on prostate cancer stage, grade, and overall recurrence risk in men undergoing radical prostatectomy. Urology. 2016;93:77-85

15. Oury F, Ferron M, Huizhen W, Confavreux C, Xu L, Lacombe J, Srinivas P, Chamouni A, Lugani F, Lejeune $\mathrm{H}$. Osteocalcin regulates murine and human fertility through a pancreas-bone-testis axis. J Clin Invest. 2013:123:2421-33.

16. Pi M, Faber P, Ekema G, Jackson PD, Ting A, Wang N, Fontilla-Poole M, Mays RW, Brunden KR, Harrington JJ. Identification of a novel extracellular cationsensing G-protein-coupled receptor. J Biol Chem. 2005;280:40201-9.

17. Pi M, Quarles LD. Novel bone endocrine networks integrating mineral and energy metabolism. Curr Osteoporos Rep. 2013;11:391-9.

18. Wei J, Hanna T, Suda N, Karsenty G, Ducy P. Osteocalcin promotes B-cell proliferation during development and adulthood through Gprc6a. Diabetes. 2014;63:1021-31.

19. Hill H, Grams J, Walton R, Liu J, Moellering D, Garvey W. Carboxylated and uncarboxylated forms of osteocalcin directly modulate the glucose transport system and inflammation in adipocytes. Horm Metab Res. 2014;46:341-7.

20. Liu M, Zhao Y, Yang F, Wang J, Shi X, Zhu X, Xu Y, Wei D, Sun L, Zhang Y. Evidence for a role of GPRC6A in prostate cancer metastasis based on casecontrol and in vitro analyses. Eur Rev Med Pharmacol Sci. 2016;20:2235-48.

21. Takata R, Akamatsu S, Kubo M, Takahashi A, Hosono N, Kawaguchi T, Tsunoda T, Inazawa J, Kamatani N, Ogawa O. Genome-wide association study identifies five new susceptibility loci for prostate cancer in the Japanese population. Nat Genet. 2010:42:751-4.

22. Long Q-Z, Du Y-F, Ding X-Y, Li X, Song W-B, Yang Y, Zhang P, Zhou J-P, Liu $X-G$. Replication and fine mapping for association of the C2orf43, FOXP4, GPRC6A and RFX6 genes with prostate cancer in the Chinese population. PLoS One. 2012;7:e37866.

23. Haiman CA, Han Y, Feng Y, Xia L, Hsu C, Sheng X, Pooler LC, Patel Y, Kolonel LN, Carter E. Genome-wide testing of putative functional exonic variants in relationship with breast and prostate cancer risk in a multiethnic population. PLoS Genet. 2013;9:e1003419.

24. Nimptsch K, Rohrmann S, Nieters A, Linseisen J. Serum undercarboxylated osteocalcin as biomarker of vitamin $\mathrm{K}$ intake and risk of prostate cancer: a nested case-control study in the Heidelberg cohort of the European prospective investigation into cancer and nutrition. Cancer Epidemiol Prev Biomarkers. 2009;18:49-56.

25. Hayashi Y, Kawakubo-Yasukochi T, Mizokami A, Takeuchi H, Nakamura S, Hirata M. Differential roles of carboxylated and uncarboxylated osteocalcin in prostate cancer growth. J Cancer. 2016;7:1605. 
26. Pi M, Kapoor K, Wu Y, Ye R, Senogles SE, Nishimoto SK, Hwang D-J, Miller DD, Narayanan R, Smith JC. Structural and functional evidence for testosterone activation of GPRC6A in peripheral tissues. Mol Endocrinol. 2015;29:1759-73.

27. Zarif JC, Miranti CK. The importance of non-nuclear AR signaling in prostate cancer progression and therapeutic resistance. Cell Signal. 2016;28:348-56.

28. Jacobsen SE, Nørskov-Lauritsen L, Thomsen ARB, Smajilovic S, Wellendorph P, Larsson NH, Lehmann A, Bhatia VK, Bräuner-Osborne H. Delineation of the GPRC6A receptor signaling pathways using a mammalian cell line stably expressing the receptor. J Pharmacol ExpTher. 2013;347:298-309.

29. Rueda P, Harley E, Lu Y, Stewart GD, Fabb S, Diepenhorst N, Cremers B, Rouillon M-H, Wehrle I, Geant A. Murine GPRC6A mediates cellular responses to $L$-amino acids, but not osteocalcin variants. PLoS One. 2016;11:e0146846

30. MacArthur DG, Balasubramanian S, Frankish A, Huang N, Morris J, Walter K, Jostins L, Habegger L, Pickrell JK, Montgomery SB. A systematic survey of loss-of-function variants in human protein-coding genes. Science. 2012;335:823-8

31. Shahabi A, Lewinger JP, Ren J, April C, Sherrod AE, Hacia JG, Daneshmand S, Gill I, Pinski JK, Fan JB. Novel gene expression signature predictive of clinica recurrence after radical prostatectomy in early stage prostate cancer patients. Prostate. 2016:76:1239-56.

32. Kinsey-Jones JS, Alamshah A, McGavigan AK, Spreckley E, Banks K, Cereceda Monteoliva N, Norton M, Bewick GA, Murphy KG. GPRC6a is not required for the effects of a high-protein diet on body weight in mice. Obesity. 2015:23:1194-200.

33. Jørgensen $S$, Have CT, Underwood CR, Johansen LD, Wellendorph P, Gjesing AP, Jørgensen CV, Quan S, Rui G, Inoue A. Genetic Variations in the Human GPRC6A Receptor Control Cell Surface Expression and Function. J Biol Chem 2017:292:1524-34.

34. Cong L, Ran FA, Cox D, Lin S, Barretto R, Habib N, Hsu PD, Wu X, Jiang W, Marraffini LA. Multiplex genome engineering using CRISPR/Cas systems. Science. 2013;339:819-23.

35. Kuang D, Yao Y, Lam J, Tsushima RG, Hampson DR. Cloning and characterization of a Family C orphan G-protein coupled receptor. J Neurochem. 2005;93:383-91.

36. Shalem O, Sanjana NE, Hartenian E, Shi X, Scott DA, Mikkelsen TS, Heckl D, Ebert BL, Root DE, Doench JG. Genome-scale CRISPR-Cas9 knockout screening in human cells. Science. 2014;343:84-7.

37. Pi M, Wu Y, Lenchik NI, Gerling I, Quarles LD. GPRC6A mediates the effects of $L$-arginine on insulin secretion in mouse pancreatic islets. Endocrinology. 2012;153:4608-15.

38. Consortium GP. A global reference for human genetic variation. Nature. 2015;526:68-74

39. Thomsen AR, Plouffe B, Cahill TJ, Shukla AK, Tarrasch JT, Dosey AM, Kahsai AW, Strachan RT, Pani B, Mahoney JP. GPCR-G protein- $\beta$-arrestin supercomplex mediates sustained G protein signaling. Cell. 2016;166:907-19.

40. Pi M, Nishimoto SK, Quarles LD. GPRC6A: Jack of all metabolism (or master of none). Molecular Metabolism. 2017;6:185-93.

41. Fujiwara T, Kanazawa S, Ichibori R, Tanigawa T, Magome T, Shingaki K, Miyata S, Tohyama M, Hosokawa K. L-arginine stimulates fibroblast proliferation through the GPRC6A-ERK1/2 and PI3K/Akt pathway. PLoS One. 2014;9:e92168.

42. Dehghan A, Dupuis J, Barbalic M, Bis JC, Eiriksdottir G, Lu C, Pellikka N, Wallaschofski H, Kettunen J, Henneman P. Meta-analysis of genome-wide association studies in $>80000$ subjects identifies multiple loci for C-reactive protein levels clinical perspective. Circulation. 2011;123:731-8.

43. Lv X, Liu J, Shi Q, Tan Q, Wu D, Skinner JJ, Walker AL, Zhao L, Gu X, Chen N. In vitro expression and analysis of the 826 human $G$ protein-coupled receptors. Protein Cell. 2016;7:325-37.

44. De Toni L, Guidolin D, De Filippis V, Tescari S, Strapazzon G, Santa Rocca M, Ferlin A, Plebani M, Foresta C. Osteocalcin and Sex hormone binding globulin compete on a specific binding site of GPRC6A. Endocrinology. 2016;157:4473-86

45. Jewell JL, Russell RC, Guan K-L. Amino acid signalling upstream of mTOR. Nat Rev Mol Cell Biol. 2013;14:133-9.

46. Kinkade CW, Castillo-Martin M, Puzio-Kuter A, Yan J, Foster TH, Gao H, Sun Y, Ouyang X, Gerald WL, Cordon-Cardo C. Targeting AKT/mTOR and ERK MAPK signaling inhibits hormone-refractory prostate cancer in a preclinical mouse model. J Clin Invest. 2008;118:3051-64.

47. Ge C, Zhao G, Li Y, Li H, Zhao X, Pannone G, Bufo P, Santoro A, Sanguedolce $F$, Tortorella S. Role of Runx2 phosphorylation in prostate cancer and association with metastatic disease. Oncogene. 2016;35:366.
48. Gupta A, Zhou CQ, Chellaiah MA. Osteopontin and MMP9: Associations with VEGF expression/secretion and angiogenesis in PC3 prostate cancer cells. Cancers. 2013;5:617-38.

49. Alimirah F, Chen J, Basrawala Z, Xin H, Choubey D. DU-145 and PC-3 human prostate cancer cell lines express androgen receptor: implications for the androgen receptor functions and regulation. FEBS Lett. 2006;580:2294-300.

50. Mostaghel EA. Steroid hormone synthetic pathways in prostate cancer. Translational Androl Urol. 2013;2:212-27.

51. Chun JY, Nadiminty N, Dutt S, Lou W, Yang JC, Kung H-J, Evans CP, Gao AC. Interleukin-6 regulates androgen synthesis in prostate cancer cells. Clin Cancer Res. 2009;15:4815-22.

52. Thulin MH, Nilsson ME, Thulin P, Céraline J, Ohlsson C, Damber J-E, Welén K. Osteoblasts promote castration-resistant prostate cancer by altering intratumoral steroidogenesis. Mol Cell Endocrinol. 2016;422:182-91.

53. Logothetis $\mathrm{CJ}$, Lin $\mathrm{S}-\mathrm{H}$. Osteoblasts in prostate cancer metastasis to bone. Nat Rev Cancer. 2005;5:21-8.

54. Yeung F, Law WK, Yeh C-H, Westendorf JJ, Zhang Y, Wang R, Kao C, Chung LW. Regulation of human osteocalcin promoter in hormone-independent human prostate cancer cells. J Biol Chem. 2002;277:2468-76.

55. Pi M, Parrill AL, Quarles LD. GPRC6A mediates the non-genomic effects of steroids. J Biol Chem. 2010;285:39953-64.

56. Dagogo-Jack S. Ethnic disparities in type 2 diabetes: pathophysiology and implications for prevention and management. J Natl Med Assoc. 2003;95:774.

57. Powell IJ. Epidemiology and pathophysiology of prostate cancer in AfricanAmerican men. J Urol. 2007:177:444-9.

\section{Submit your next manuscript to BioMed Central and we will help you at every step:}

- We accept pre-submission inquiries

- Our selector tool helps you to find the most relevant journal

- We provide round the clock customer support

- Convenient online submission

- Thorough peer review

- Inclusion in PubMed and all major indexing services

- Maximum visibility for your research

Submit your manuscript at www.biomedcentral.com/submit
) Biomed Central 